\title{
Experimental Organism Aborted
}

National Cancer Institute

\section{Source}

National Cancer Institute. Experimental Organism Aborted. NCI Thesaurus. Code

C124616.

Loss of all or part of the products of conception prior to the scheduled c-section date or expected delivery date. 